\title{
Oral butyrate does not affect innate immunity and islet autoimmunity in individuals with longstanding type 1 diabetes: a randomised controlled trial
}

\author{
Pieter F. de Groot ${ }^{1}$ - Tatjana Nikolic ${ }^{2}$ - Sultan Imangaliyev ${ }^{1}$. Siroon Bekkering ${ }^{1,3}$ - Gaby Duinkerken ${ }^{2}$ - Fleur M. Keij ${ }^{2}$. \\ Hilde Herrema ${ }^{1}$. Maaike Winkelmeijer ${ }^{1}$. Jeffrey Kroon ${ }^{1}$ • Evgeni Levin ${ }^{1}$ - Barbara Hutten ${ }^{4}$ - Elles M. Kemper ${ }^{5}$. \\ Suat Simsek ${ }^{6}$ - Johannes H. M. Levels ${ }^{1}$ • Flora A. van Hoorn ${ }^{1}$ • Renuka Bindraban ${ }^{1}$. Alicia Berkvens ${ }^{1}$. \\ Geesje M. Dallinga-Thie ${ }^{1} \cdot$ Mark Davids $^{1}$ • Frits Holleman ${ }^{1}$ • Joost B. L. Hoekstra ${ }^{1}$ • Erik S. G. Stroes ${ }^{1} \cdot$ Mihai Netea $^{3,7}$. \\ Daniël H. van Raalte ${ }^{1,8} \cdot$ Bart O. Roep $^{2,9} \cdot$ Max Nieuwdorp ${ }^{1,8}$
}

Received: 21 August 2019 / Accepted: 6 November 2019 / Published online: 8 January 2020

(C) Springer-Verlag GmbH Germany, part of Springer Nature 2020

\begin{abstract}
Aims/hypothesis The pathophysiology of type 1 diabetes has been linked to altered gut microbiota and more specifically to a shortage of intestinal production of the short-chain fatty acid (SCFA) butyrate, which may play key roles in maintaining intestinal epithelial integrity and in human and gut microbial metabolism. Butyrate supplementation can protect against autoimmune diabetes in mouse models. We thus set out to study the effect of oral butyrate vs placebo on glucose regulation and immune variables in human participants with longstanding type 1 diabetes.

Methods We administered a daily oral dose of $4 \mathrm{~g}$ sodium butyrate or placebo for 1 month to 30 individuals with longstanding type 1 diabetes, without comorbidity or medication use, in a randomised $(1: 1)$, controlled, double-blind crossover trial, with a washout period of 1 month in between. Participants were randomly allocated to the 'oral sodium butyrate capsules first' or 'oral placebo capsules first' study arm in blocks of five. The clinical investigator received blinded medication from the clinical trial pharmacy. All participants, people doing measurements or examinations, or people assessing the outcomes were blinded to group assignment. The primary outcome was a change in the innate immune phenotype (monocyte subsets and in vitro cytokine production). Secondary outcomes were changes in blood markers of islet autoimmunity (cell counts, lymphocyte stimulation indices and CD8 quantum dot assays), glucose and lipid metabolism, beta cell function (by mixed-meal test), gut microbiota and faecal SCFA. The data was collected at the Amsterdam University Medical Centers.

Results All 30 participants were analysed. Faecal butyrate and propionate levels were significantly affected by oral butyrate supplementation and butyrate treatment was safe. However, this modulation of intestinal SCFAs did not result in any significant
\end{abstract}

Electronic supplementary material The online version of this article (https://doi.org/10.1007/s00125-019-05073-8) contains peer-reviewed but unedited supplementary material, which is available to authorised users.

Pieter F. de Groot

p.f.degroot@amsterdamumc.nl

1 Department of Internal and Vascular Medicine, Academic Medical Center, Meibergdreef 9, Room D3-316, 1105 AZ Amsterdam, the Netherlands

2 Department of Immunohematology and Blood Transfusion, Leiden University Medical Center, Leiden, the Netherlands

3 Department of Internal Medicine, Radboud University Medical Center, Nijmegen, the Netherlands

4 Department of Epidemiology, Amsterdam University Medical Centers, Academic Medical Centre, Amsterdam, the Netherlands

5 Clinical Pharmacy, Amsterdam University Medical Centers, Academic Medical Centre, Amsterdam, the Netherlands

6 Department of Internal Medicine, Alkmaar Medical Center (MCA), Alkmaar, the Netherlands

7 Department for Genomics \& Immunoregulation, Life and Medical Sciences Institute (LIMES), University of Bonn, Bonn, Germany

8 Diabetes Center, Department of Internal Medicine, Amsterdam University Medical Centers, VU University Medical Centre, Amsterdam, the Netherlands

9 Department of Diabetes Immunology, Diabetes \& Metabolism Research Institute at the Beckman Research Institute, City of Hope, Duarte, CA, USA 


\section{Research in context}

What is already known about this subject?

- Type 1 diabetes is associated with gut microbiota and short-chain fatty acid metabolism

- Butyrate is an immunomodulator in autoimmune diabetes

- Butyrate and acetate supplementation can alleviate autoimmune diabetes in NOD mice

What is the key question?

- Can butyrate supplementation modulate the immune system towards immune tolerance to beta cell antigens in individuals with type 1 diabetes?

What are the new findings?

- Contrary to expectations, butyrate supplementation, in a sufficient dose to modulate intestinal butyrate levels vs placebo, did not affect the phenotype or function of any of a large number of innate or adaptive immune cells tested, or any clinical variable measured, in participants with type 1 diabetes

How might this impact on clinical practice in the foreseeable future?

- Our data do not support the use of short-chain fatty acid supplementation to modulate the immunological tone in those with longstanding type 1 diabetes mellitus

changes in adaptive or innate immunity, or in any of the other outcome variables. In our discussion, we elaborate on this important discrepancy with previous animal work.

Conclusions/interpretation Oral butyrate supplementation does not significantly affect innate or adaptive immunity in humans with longstanding type 1 diabetes.

Trial registration Netherlands Trial Register: NL4832 (www.trialregister.nl).

Data availability Raw sequencing data are available in the European Nucleotide Archive repository (https:/www.ebi.ac.uk/ena/ browse) under study PRJEB30292.

Funding The study was funded by a Le Ducq consortium grant, a CVON grant, a personal ZONMW-VIDI grant and a Dutch Heart Foundation grant.

Keywords Butyrate $\cdot$ Diabetes $\cdot$ Microbiota $\cdot$ Short-chain fatty acids

\author{
Abbreviations \\ CCR C-C chemokine receptor \\ CRP C-reactive protein \\ CXCR CXC chemokine receptor \\ DRiP Defective ribosomal product \\ IA Islet antigen \\ IMDM Iscove's modified Dulbecco's medium \\ IQR Interquartile range \\ LPS Lipopolysaccharide \\ MFI Mean fluorescence intensity \\ NK Natural killer \\ PBMC Peripheral blood mononuclear cell \\ PPI Preproinsulin \\ SCFA Short-chain fatty acid \\ Treg T regulatory cell
}

\section{Introduction}

Type 1 diabetes is the second most frequent autoimmune disease in childhood and its incidence has tripled in the last 30 years [1]. The gut microbiota composition and, more specifically, a decrease in producers of the short-chain fatty acid (SCFA) butyrate have been implicated in type 1 diabetes development [2-6]. We and others recently reported subtle differences in the microbiota composition of a cohort of individuals with type 1 diabetes compared with control participants, including lower faecal butyryl-acetyl-CoA transferase gene activity, lower levels of butyrate-producing Roseburia and lower plasma SCFA concentrations [7]. In addition to being the main energy source for colonocytes, the SCFA butyrate has beneficial effects on markers of immunity that have 
been linked to the pathophysiology of type 1 diabetes. For example, its immune-dampening effects on lipopolysaccharide (LPS)-induced inflammation and oxidative stress have already been well-established in inflammatory bowel disease [8]. Recently, oral butyrate was found to decrease cytokine release in individuals with the metabolic syndrome and to decrease induction of trained immunity [9].

Butyrate may be beneficial in type 1 diabetes through various mechanisms. First, butyrate induces differentiation of $\mathrm{T}$ regulatory cells (Tregs) through histone $\mathrm{H} 3$ acetylation in animal models $[10,11]$, while it induces apoptosis of murine proinflammatory T cells in vitro [12]. These effects may play a role in type 1 diabetes, as specific autoreactive $\mathrm{T}$ cells have been found to be primed in the gut of NOD mice [13]. Finally, increased butyrate production via co-fermentation of acetate by communities rich in Akkermansia may protect against the development of anti-islet cell autoantibodies [14]. A recent study showed that a diet rich in butyrate and acetate can prevent type 1 diabetes in NOD mice [15] and confirmed that high acetate and butyrate levels coincide with protection from autoimmune diabetes in NOD mice, as was previously reported [16]. In contrast, intestinal microbes will start degrading the protective mucus layer in order to maintain SCFA production if deprived of dietary fibre, thereby increasing bacterial translocation and immunogenicity [17]. These changes are associated with increased circulating levels of toll-like receptor 2 and 4 ligands in type 1 diabetes, due to increased intestinal permeability $[18,19]$. In addition, butyrate supplementation induces hyporesponsiveness to LPS, which coincides with less LPS-induced IL-6 and IL-12 secretion in intestinal macrophages, eliciting tolerance towards intestinal microbiota [20]. Furthermore, animal studies have suggested that butyrate can improve intestinal barrier function by upregulating tightjunction proteins [21]. Finally, the interaction between gut microbes and the innate immune system has been shown to be a crucial factor in the development of autoimmune diabetes in NOD mice [22].

Insulitis and beta cell destruction are focal and nonexhaustive processes [23], and most individuals with longstanding type 1 diabetes retain some residual C-peptide secretion [24, 25]. In line with this, it has been postulated that human type 1 diabetes beta cells are in a state of senescence [26] that might be reversed by restoring the intestinal immune balance (e.g. by restoring the SCFA balance). As the innate immune system has now been established to retain immunological memory after previous encounters with inflammatory stimuli [27] such as gut microbial antigens ('trained innate immunity'), the SCFAs, including butyrate, may be excellent candidates to downregulate such a proinflammatory status in type 1 diabetes. Therefore, we studied the effect of oral butyrate capsule supplementation compared with placebo for 4 weeks on both safety and the innate and adaptive immune systems in individuals with longstanding type 1 diabetes. Moreover, we studied the effects on islet autoimmunity and (mixed-meal test stimulated) residual beta cell function in relation to changes in intestinal microbiota composition.

\section{Methods}

Participant recruitment Participants with type 1 diabetes were recruited from outpatient diabetes clinics in the Amsterdam region between February 2015 and February 2016. All participants provided written informed consent and all study procedures were approved by the institutional review board (ethics committee) of the Academic Medical Center and conducted in accordance with the Declaration of Helsinki. The study was prospectively registered with the Netherlands Trial Register (NL4832). Inclusion criteria for participants were a diagnosis of type 1 diabetes, European descent, age 18-65 years and normal BMI $\left(18.5-25.0 \mathrm{~kg} / \mathrm{m}^{2}\right)$. Exclusion criteria were known determinants of an altered microbiota composition, including medication use besides insulin (e.g. statins, proton-pump inhibitors), use of antibiotics in the last 3 months and use of probiotics. In addition, individuals with unusual dietary habits (including a vegetarian diet) and medical conditions beside type 1 diabetes that are believed to affect or associate with glucose metabolism or an altered gut microbiota (e.g. coeliac disease, cholecystectomy, irritable bowel syndrome, autoimmune thyroid disease) [28] were excluded.

Randomisation and study design Participants were randomly allocated to the 'oral sodium butyrate capsules first' or 'oral placebo capsules first' study arm in blocks of five. The clinical investigator received blinded medication from the clinical trial pharmacy. This was dispersed to the participants, who started with either $4 \mathrm{~g}$ ( $2 \mathrm{~g}$ twice daily) sodium butyrate or placebo capsules (produced by Sensilab, Lódz, Poland). Of note, 4 g/ day was the maximum daily dose allowed by the institutional review board based on previous human intervention studies [29, 30]. Compliance was evaluated by counting the number of capsules returned after 4 weeks of treatment. A washout period of 4 weeks was maintained before transfer to the other study arm. The study and statistical analysis scheme is shown in electronic supplementary material (ESM) Fig. 1. Participants were asked to maintain their habitual physical activity pattern, but to refrain from heavy exercise in the days preceding study visits. Participants were encouraged to continue with their usual diet. All participants completed an online nutritional diary (https://mijn.voedingscentrum.nl/nl/eetmeter) for 1 week 
before each study visit to monitor their intake of carbohydrates, fat, protein and fibre.

Study procedures During each study visit, anthropometric measurements (i.e. height, weight and hip and waist circumference) were taken and BP was measured. Blood was sampled after an overnight fast. Plasma was isolated by centrifugation at $200 \mathrm{~g}$ for $15 \mathrm{~min}$ at $4^{\circ} \mathrm{C}$ and plasma aliquots were stored for further analyses at $-80^{\circ} \mathrm{C}$. Urine was collected for $24 \mathrm{~h}$ prior to each visit. Faecal samples were collected at home on the morning of the visit and frozen at $-80^{\circ} \mathrm{C}$ at the research facility, or on the night before the visit and frozen at home, stored in a freezer $\left(-20^{\circ} \mathrm{C}\right)$ overnight and transferred frozen to the clinical research unit.

Biochemistry Fasting plasma glucose concentrations and Creactive protein (CRP) (Roche, Basel, Switzerland) were determined in plasma samples. C-peptide was measured by RIA (Millipore, Amsterdam, the Netherlands), while total cholesterol, HDL-cholesterol and triacylglycerols were determined in plasma using commercially available enzymatic assays (Randox, Antrim, UK; and DiaSys, Holzheim, Germany). All analyses were performed using a Selectra autoanalyser (Sopachem, Ochten, the Netherlands). LDLcholesterol was calculated using the Friedewald formula. Calprotectin was determined in faeces using a commercial ELISA (Bühlmann, Schönenbuch, Switzerland).

\section{Mixed-meal test stimulated C-peptide test (for residual beta} cell function) For the mixed-meal test stimulated C-peptide test, participants did not use their long-acting exogenous insulin injections and used only half of their evening dose of shortacting insulin on the evening before each mixed-meal test, as previously described [31]. After an overnight fast and without taking their short-acting morning insulin dose, a mixed-meal test was performed with $6 \mathrm{ml} / \mathrm{kg}$ Sustacal Boost (Nestlé, Nunspeet, the Netherlands) with a maximum of $360 \mathrm{ml}$ per person, as previously described $(1004 \mathrm{~kJ}, 6 \mathrm{~g}$ fat, $20 \mathrm{~g}$ protein, 28 g carbohydrate) [32]. Subsequent blood samples to measure C-peptide were taken at 0 and $90 \mathrm{~min}$.

Isolation of peripheral blood mononuclear cells Peripheral blood mononuclear cells (PBMCs) were isolated using Ficoll-density gradient centrifugation (Ficoll $5.7 \%$, amidotrizoate 9\%; LUMC Pharmacy, Leiden, the Netherlands) at the study site. Blood, collected in EDTAcontaining tubes, was transferred to a $50 \mathrm{ml}$ tube and 1:1 diluted with PBS (Braun, Kronberg, Germany) until a final volume of $37.5 \mathrm{ml}$. This was layered on top of $12.5 \mathrm{ml}$ Ficoll. After centrifuging, the interphase containing PBMCs was harvested and washed three times using PBS. PBMCs were resuspended in a mixture containing $2 \mathrm{ml}$ Iscove's modified Dulbecco's medium (IMDM; Lonza, Basel, Switzerland), L- glutamine, penicillin-streptomycin and $15 \%$ human serum (Sanquin, Leiden, the Netherlands), and counted.

Ex vivo PBMC stimulation experiments Monocytes were isolated and stimulated as previously described [33]. Next, the monocyte-containing layer was aspirated, washed and then plated into polystyrene 96 -well plates $\left(5 \times 10^{5} \mathrm{PBMCs}\right.$ $\mathrm{ml}^{-1}$ well ${ }^{-1}$; Corning, New York, NY, USA) for $1 \mathrm{~h}$ at $37^{\circ} \mathrm{C}$ and $5 \% \mathrm{CO}_{2}$. Non-adherent cells were removed by washing three times with warm PBS. The adhered monocytes were subsequently cultured in RPMI 1640 medium with GlutaMAX (Life Technologies/Invitrogen, Breda, the Netherlands). Finally, monocytes were stimulated for $24 \mathrm{~h}$ with medium alone (RPMI), $10 \mu \mathrm{g} / \mathrm{ml}$ Pam3Cys or $10 \mathrm{ng} / \mathrm{ml}$ LPS. After incubation, supernatants were collected and stored at $-80^{\circ} \mathrm{C}$. Cytokine production was measured by ELISAs according to the manufacturer's instructions (R\&D Systems, Minneapolis, MN, USA), as previously described [33].

Flow cytometric analysis of monocytes Erythrocytes in EDTA-containing blood were lysed using erythrocyte-lysis buffer (Affymetrix; eBioscience, San Diego, CA, USA), after which the cells were washed with PBS $\left(5 \mathrm{~min}\right.$ at $\left.4^{\circ} \mathrm{C}\right)$. Next, the supernatant containing the lysed erythrocytes was removed and leucocytes were incubated with fluorescently labelled antibodies (see ESM Table 1). Cells were washed with PBS and samples were analysed by flow cytometry (BD FACSCanto II; Becton Dickinson, Franklin Lakes, NJ, USA). Monocytes were classified according to their CD14, CD16 and HLA-DR expression. Mean fluorescence intensity (MFI) was calculated by subtracting the MFI of the isotype control from that of the marker. Data were analysed using FlowJo software 9.0 (TreeStar, Ashland, OR, USA) using the gating strategy shown in ESM Fig. 2 a.

Adaptive (T cell function) immunity Blood samples were kept at room temperature and transferred from the study site in Amsterdam to the Leiden University Medical Center and processed within $24 \mathrm{~h}$ of sampling. PBMCs were used to measure the immune response. Granulocytes were isolated for DNA extraction and genetic barcoding (HLA typing).

Lymphocyte stimulation test The lymphocyte stimulation test measures proliferation of T cells by incorporating ${ }^{3} \mathrm{H}$-thymidine in the cell's DNA. Fresh PBMCs were cultured in 96well round-bottom plates in IMDM and $10 \%$ inactivated human serum (Sanquin, Amsterdam, the Netherlands). Cells were incubated in the conditioned medium alone or in the presence of the autoantigen proteins GAD65, preproinsulin (PPI), islet antigen (IA)-2 and defective ribosomal product of proinsulin mRNA (DRiP-C-H/DRiP-R-P). All antigens were generated as recombinant protein on site and used at a final concentration of $10 \mu \mathrm{l} / \mathrm{ml}$. For controls, cells were 
stimulated with IL-2 or tetanus toxoid. Cells were incubated for 5 days, after which ${ }^{3} \mathrm{H}$-thymidine $(50 \mu \mathrm{l}, 370 \mathrm{GBq} / \mathrm{ml})$ was added for the last $18 \mathrm{~h}$. Cells were harvested on filters using an automated harvester. Proliferation was determined by measuring ${ }^{3} \mathrm{H}$-thymidine incorporation in an automatic liquid scintillation counter. All results were calculated as mean cpm in the presence of antigen and compared with medium alone. The stimulation index was calculated as mean $\mathrm{cpm}_{\text {antigen }} /$ mean $\mathrm{cpm}_{\text {medium }}$, and a stimulation index $\geq 3$ was considered a positive response.

FACS analyses and quantification of autoreactive CD8 T cells using quantum dots For phenotyping and quantification of autoreactive $\mathrm{CD}^{+} \mathrm{T}$ cells, PBMCs were stained with fluorescent antibodies according to a standard protocol as described previously [34]. Stained cells were measured using FACSCanto (phenotyping) and LSR II (quantum dot) machines (Becton Dickinson, Franklin Lakes, NJ, USA). Phenotyping data were analysed with FlowJo software (TreeStar) using the gating strategy (ESM Fig. 2b) or as described previously for quantum dot analyses [34].

Faecal SCFA concentrations Fresh morning faecal samples were collected and stored in $-80^{\circ} \mathrm{C}$ until use. SCFAs were measured using HPLC UV as previously published [30, 35].

Gut microbiota analyses Total genomic DNA was isolated from $250 \mathrm{mg}$ of faeces using an adapted repeated beadbeating method based on a previously described protocol [36]. Faecal samples were placed in bead-beating tubes and extracted twice in lysis buffer (STARbuffer, Roche, Basel, Switzerland) with bead-beating at $5.5 \mathrm{~m} / \mathrm{s}$ for $60 \mathrm{~s}$, three times in $20 \mathrm{~s}$ intervals in a Precellys 24 homogeniser (Bertin, Montigny-le-Bretonneux, France). After each bead-beating cycle, samples were heated to $95^{\circ} \mathrm{C}$ for $15 \mathrm{~min}$ and then centrifuged at full speed for $5 \mathrm{~min}$ at $4^{\circ} \mathrm{C}$. Supernatants from the two extractions were pooled and purified using the Maxwell RSC Blood DNA Kit (Promega, Madison, WI, USA). Amplicons containing the barcoded V4 region of $16 \mathrm{~S}$ genes were amplified using an adapted one-step PCR method [37]. Forward index primer (Illumina [San Diego, CA, USA] Adapter, Index, pad, link, 16Sf) and reverse index primer (Illumina Adapter, Index, pad, link, 16Sr) were used [38].

Template DNA (20 ng) was used in the PCR mix: $6 \mu \mathrm{l} 5 \times$ high-fidelity buffer (Thermo Fisher Scientific, Waltham, MA, USA), $0.75 \mu \mathrm{l}$ PCR grade nucleotide Mix (10 $\mu \mathrm{mol} / \mathrm{l}$; Thermo Fisher Scientific), $0.3 \mu$ l Phusion DNA polymerase $(2 \mathrm{U} / \mu \mathrm{l})$, $18.95 \mu \mathrm{l}$ nuclease-free water, $1.5 \mu \mathrm{l}$ forward index primer $(10 \mu \mathrm{mol} / \mathrm{l})$ and $1.5 \mu \mathrm{l}$ reverse index primer $(10 \mu \mathrm{mol} / \mathrm{l})$. The amplification program featured initial denaturation at $98^{\circ} \mathrm{C}$ for $30 \mathrm{~s}, 25$ cycles of denaturation at $98^{\circ} \mathrm{C}$ for $10 \mathrm{~s}$, annealing at $55^{\circ} \mathrm{C}$ for $20 \mathrm{~s}$, elongation at $72^{\circ} \mathrm{C}$ for $90 \mathrm{~s}$ and extension at $72^{\circ} \mathrm{C}$ for $10 \mathrm{~min}$ [38]. PCR was performed using a Biometra thermal cycler (Göttingen, Germany). To confirm the presence of the PCR product, $1 \%$ agarose gel electrophoresis containing ethidium bromide was used. PCR products were purified using the Biomek FX robot (Beckman Coulter, Brea, CA, USA) with AMPure XP beads (Beckman Coulter). Amplicons were quantified using the Qubit dsDNA BR Assay Kit (Thermo Fisher). The samples, each labelled with a unique index, were pooled in equal molar concentrations. The quality and quantity of the pooled library was checked using a High Sensitivity DNA chip (Agilent, Santa Clara, CA, USA) on the Bioanalyzer 2100 (Agilent) and Qubit dsDNA BR Assay Kit. The libraries were sequenced using a MiSeq platform (Illumina, San Diego, CA, USA) using V3 chemistry with $2 \times 251$ cycles. Forward and reverse reads were length trimmed at 240 and $210 \mathrm{bp}$, respectively, and amplicon sequence variants were inferred and merged using dada2 (https://benjjneb.github.io/dada2/dada-installation. html) v1.5.2. Taxonomy was assigned using dada2 implementation of the RDP classifier and SILVA $16 \mathrm{~S}$ ribosomal database V128 (https://www.arb-silva.de/ documentation/release-128/). Microbiota data were further analysed and visualised using phyloseq (https:// bioconductor.org/packages/release/bioc/html/phyloseq.html), vegan (https://cran.r-project.org/web/packages/vegan/index. html) and picante (https://cran.r-project.org/web/packages/ picante/index.html), and analyses were performed as previously published [39]. Raw sequencing data were submitted to the European Nucleotide Archive repository under study PRJEB30292. A microbiota amplicon sequence variant abundance table is supplied in the ESM, paired with the taxonomy key (ESM Tables 2, 3).

Study endpoints The primary endpoint was a change in innate immune function (peripheral monocyte phenotype analysed by flow cytometry and in vitro monocyte cytokine production) with butyrate vs placebo treatment. Secondary endpoints were changes in adaptive immunological variables (i.e. peripheral $\mathrm{T}$ cell subsets, residual beta cell function, $\mathrm{HbA}_{1 \mathrm{c}}$, total daily insulin dose, markers of intestinal and systemic inflammation [faecal calprotectin, CRP, leucocytes]) as well as changes in SCFA concentrations and gut microbiome composition following sodium butyrate vs placebo treatment.

Power calculation and statistics We based our sample-size calculation on the assumption that 4 weeks of oral butyrate treatment would induce a $30 \%$ reduction in in vitro (LPS) stimulated monocyte TNF- $\alpha$ secretion (from 2.000 to $1400 \mathrm{pg} / \mathrm{ml}$ with an $\mathrm{SD}$ of $500 \mathrm{pg} / \mathrm{ml}$ ), whereas no effect was expected with placebo. Using a $t$ test on group means, a 0.05 significance level and power of 0.8 , the necessary sample size was 30 . Three participants were replaced after retracting their consent for private reasons (respectively: too burdensome, other obligations and anxiety problems). The study ended in January 2017. 
Table $1 \quad$ Baseline and follow-up characteristics

\begin{tabular}{|c|c|c|c|c|c|c|}
\hline Baseline and follow-up characteristics & Before butyrate & After butyrate & $p$ value $^{\mathrm{a}}$ & $\begin{array}{l}\text { Absolute change } \\
\text { butyrate }\end{array}$ & $\begin{array}{l}\text { Absolute change } \\
\text { placebo }\end{array}$ & $p$ value of $\Delta \mathrm{s}^{\mathrm{b}}$ \\
\hline Age (median years, min-max) & $32.5(22-61)$ & & & & & \\
\hline Male, $n /$ total & $20 / 30$ & & & & & \\
\hline Diabetes duration, years (IQR) & $8(4-16)$ & & & & & \\
\hline Anti-GAD65 positive, $n /$ total & $18 / 30$ & & & & & \\
\hline Anti-IA-2 positive, $n /$ total & $14 / 30$ & & & & & \\
\hline GAD65 and IA- 2 negative, $n /$ total & $8 / 30$ & & & & & \\
\hline BMI, $\mathrm{kg} / \mathrm{m}^{2}$ & $23.5(21.4-25.6)$ & $23.5(21.5-25.5)$ & NS & 0 & 0 & NS \\
\hline Daily insulin use, $\mathrm{U} / \mathrm{kg}$ & $0.59(0.33-0.85)$ & $0.61(0.35-0.89)$ & NS & 0 & 0 & NS \\
\hline $\mathrm{HbA} \mathrm{A}_{1 \mathrm{c}}, \mathrm{mmol} / \mathrm{mol}$ & $55(45-65)$ & $55(41-70)$ & NS & 0 & -2 & NS \\
\hline $\mathrm{HbA}_{1 \mathrm{c}}, \%$ & $7.3(5.9-8.6)$ & $7.3(5.4-9.3)$ & NS & 0 & -0.2 & NS \\
\hline $\begin{array}{l}\text { Stimulated C-peptide AUC, } \\
\mathrm{mmol} / 1 \times \min \end{array}$ & $163(-256-583)$ & $155(-125-434)$ & NS & -11 & +4 & NS \\
\hline HDL-cholesterol, mmol/1 & $1.46(1.04-1.88)$ & $1.51(1.00-2.01)$ & NS & +0.06 & -0.08 & NS \\
\hline LDL-cholesterol, mmol/1 & $2.65(1.76-3.53)$ & $2.80(1.83-3.77)$ & NS & +0.15 & -0.19 & NS \\
\hline Triacylglycerols, mmol/1 & $0.69(0.28-1.10)$ & $0.77(0.28-1.25)$ & NS & +0.08 & +0.01 & NS \\
\hline $\mathrm{CRP}, \mathrm{nmol} / 1$ & $5.7(-6.0-17.4)$ & $5.7(-2.1-13.6)$ & NS & 0 & +1 & NS \\
\hline Faecal calprotectin, $\mu \mathrm{g} / \mathrm{g}$ & $25(-3-53)$ & $22(-15-59)$ & NS & -3 & -3 & NS \\
\hline Faecal acetate, $\mu \mathrm{mol} / \mathrm{g}$ & $103(47-160)$ & $87(47-128)$ & 0.031 & -16 & -1.4 & NS \\
\hline Faecal propionate, $\mu \mathrm{mol} / \mathrm{g}$ & $23.4(5.7-41.0)$ & $18.6(4.5-32.7)$ & 0.003 & -4.7 & +0.57 & 0.02 \\
\hline Faecal butyrate, $\mu \mathrm{mol} / \mathrm{g}$ & $19.9(-5.6-45.4)$ & $13.7(7.9-35)$ & 0.001 & -6.2 & +5.3 & 0.03 \\
\hline Total faecal SCFA, $\mu \mathrm{mol} / \mathrm{g}$ & $168(84-253)$ & $145(69-223)$ & 0.014 & -23 & +3.5 & NS $(0.09)$ \\
\hline Total energy intake, $\mathrm{kJ} /$ day & $7880(5633-10,118)$ & $7750(5717-9771)$ & NS & -130 & -100 & NS \\
\hline Protein intake, $g /$ day & $76(51-102)$ & $74(59-89)$ & NS & -2 & +5 & NS \\
\hline Carbohydrate intake, $g$ /day & $192(108-275)$ & $198(127-269)$ & NS & +6 & +4 & NS \\
\hline Fat intake, $g /$ day & $77(62-92)$ & $76(61-92)$ & NS & -1 & +5 & NS \\
\hline Fibre intake, $g$ /day & $20(9-30)$ & $20(12-28)$ & NS & 0 & +1 & NS \\
\hline
\end{tabular}

Data are expressed as medians with IQR except where otherwise specified (i.e. $n /$ total or median [min-max])

$p$ values were calculated using Mann-Whitney test

${ }^{a} p$ values comparing before vs after butyrate

${ }^{\mathrm{b}} p$ values comparing $\Delta$ butyrate vs $\Delta$ placebo

A summary of the analysis methods is provided in ESM Fig. 1b. For baseline differences between groups and to compare before vs after butyrate, a paired two-sided Wilcoxon's rank sum test or Student's $t$ test was used depending on the distribution of the data. Accordingly, data are expressed as means \pm SEM or medians with interquartile range (IQR). Spearman's Rank test was used for correlation analyses. To compare differences between the treatment groups, a Wilcoxon's test was performed on the $\Delta$ butyrate and $\Delta$ placebo. Then, to assess a 'carry-over' effect, an unpaired two-sided Student's $t$ test was performed between the butyrate-first group and the placebo-first group. 'Carry-over' effects were defined as late effects in the butyratefirst group $(n=15)$ that were registered in the placebo phase, but that may be attributed to prior butyrate use.

\section{Results}

Thirty participants with type 1 diabetes were included; 15 were allocated to the 'oral sodium butyrate capsules first' and 15 to the 'oral placebo capsules first' study arm. Participants had a median age of 32.5 years, with a mean diabetes duration of 8 years; $67 \%$ were male. Macronutrient intake did not differ between study periods. Other baseline and follow-up characteristics can be found in Table 1.

Innate immune system phenotype and functional assays Monocyte subset analysis showed a significant numerical increase in CD11b expression upon sodium butyrate administration (Fig. 1k), but this was not statistically significant when compared with the change after placebo $(p=0.32)$. Ex vivo cytokine production (TNF- $\alpha$, IL-10 and IL- $1 \beta$ ) did not show significant changes after stimulation with either LPS or Pam3Cys (Fig. 2).

Adaptive immune system phenotype and T cell functional in vitro assays Islet autoreactive $\mathrm{CD} 8 \mathrm{~T}$ cells were enumerated using quantum dot multiplexed HLA-A2 tetramers loaded with a range of islet epitopes (Fig. 3). IA-2-specific $\mathrm{CD} 8^{+} \mathrm{T}$ cells were significantly decreased upon oral butyrate administration $(p=0.009)$, which persisted when compared with the change after placebo $(p=0.01)$ and no carry-over effect was observed (Fig. 3d). It should be noted, however, that (in contrast to all other analyses, which were performed using data from all 30 participants) quantum dot analyses could only be performed in 13/30 people (those with HLA-DR3/4 and 
Fig. 1 FACS data of monocyte subsets. MFI is reported as the median with IQR. Paired twosided Wilcoxon's ranked sum tests were used to assess significance: $* p<0.05$. The gating strategy can be found in ESM Fig. 2 and its legend. All participants were included in this analysis. Whiskers show minimum and maximum values (other than outliers, shown as dots). AB, after butyrate; AP, after placebo; $\mathrm{BB}$, before butyrate; $\mathrm{BP}$, before placebo; TLR, toll-like receptor
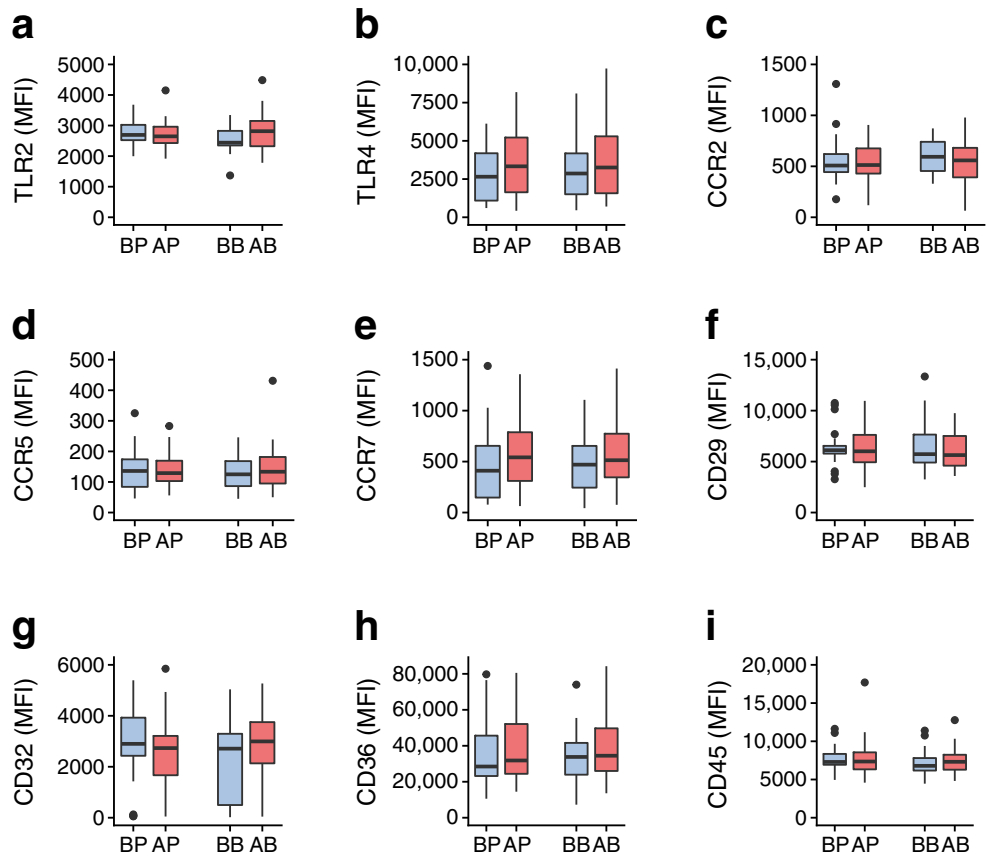

h
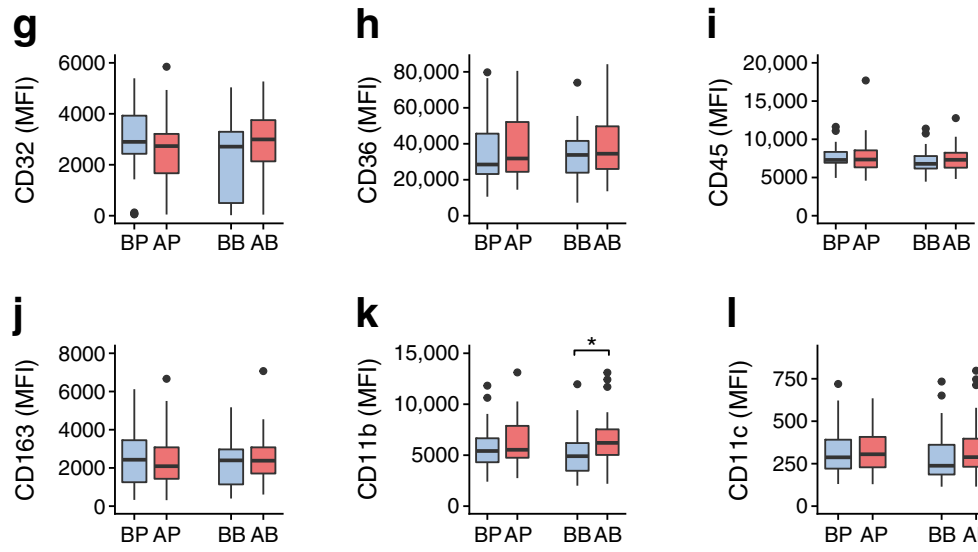

k

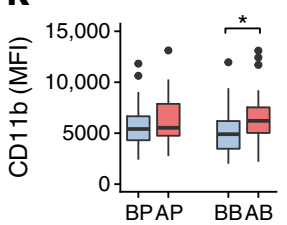

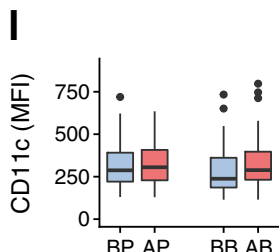

-DQ2/8) and that a large baseline difference was seen between the butyrate and placebo treatment groups. CD8 cells specific for other epitopes did not change significantly. Lymphocyte stimulation assays showed no statistically significant changes in T cell proliferation in response to islet autoantigens (Fig. 4). Flow cytometry analysis showed no significant change in natural killer (NK) cells, B cells, $\beta 7 / \mathrm{CD} 49 \mathrm{~d}$ and CXC chemokine receptor (CXCR)3/C-C chemokine receptor (CCR)5positive $\mathrm{CD}^{+} \mathrm{T}$ cells, $\beta 7 / \mathrm{CD} 49 \mathrm{~d}$ and $\mathrm{CXCR} 3 / \mathrm{CCR} 5 \mathrm{CD}^{+}$ T cells or natural Tregs (Fig. 5), or several additional lymphocyte subsets (ESM Fig. 3).

SCFAs, glucose metabolism and inflammatory variables In line with our previous work, in which oral butyrate was administered to participants with the metabolic syndrome [30], faecal SCFA content decreased significantly after 4 weeks of oral butyrate treatment (Fig. 6f), with a concomitant reduction in faecal levels of acetate, propionate and butyrate (Table 1; Fig. 6g-i).

No effects on weight, BMI, energy intake, fasting glucose or total daily insulin dose were seen (Table 1), nor on inflammatory variables (plasma CRP levels and faecal calprotectin) (Fig. 6d, e). Finally, while 21 out of 30 participants still had some endogenous C-peptide secretion at baseline before the mixed-meal test, as previously described in individuals with longstanding type 1 diabetes [24, 25], residual beta cell function (mixed-meal test-stimulated C-peptide levels) or $\mathrm{HbA}_{1 \mathrm{c}}$ did not improve significantly (Fig. 6a, b), which was in line with our expectations from this short treatment course.

Gut microbiota composition upon oral butyrate treatment By applying the Extreme Gradient Boosting classification algorithm [40] in combination with a stability selection procedure [41] to identify which intestinal bacterial species were most discriminative between oral butyrate vs placebo treatment, we identified Lachnospira pectinoschiza, Ruminococcaceae spp., Marvinbryantia spp., Erysipelotrichaceae spp., Bifidobacterium adolescentis/faecale/stercoris, Dorea formicigenerans and several Lachnospiraceae spp. to be the top ten most discriminative taxa (Fig. 7a). A more complete list including the top 30 features that were most discriminatory is provided in ESM Fig. 4. The receiver-operator curve AUC was $0.63 \pm 0.15$ on the test dataset. The observed discriminative effect of the selected top ten taxa on separation between butyrate and placebo can be appreciated in the partial leastsquares discriminant analysis plot (Fig. 7c), showing poor separation of the butyrate and placebo groups. Finally, faecal microbiota diversity, as calculated using the Shannon index (alpha diversity), did not alter significantly during the study period in either group (Shannon's diversity index: butyrate, 
Fig. 2 Ex vivo monocyte production of three different cytokines (IL-10, TNF- $\alpha$ and IL$1 \beta$ ) after stimulation with two toll-like receptor ligands (LPS and Pam3Cys). RPMI served as the negative control (data not shown). Data are means \pm SEM, with the dots and connecting lines representing individual

participants. A few samples could not be analysed because too little material was obtained: two values were missing from the "before placebo' period, two values from the 'before butyrate' group and one value from the 'after butyrate' group; therefore this analysis is based on $28 / 30$ placebo vs $27 / 30$ butyrate. $\mathrm{AB}$, after butyrate; $\mathrm{AP}$, after placebo; $\mathrm{BB}$, before butyrate; $\mathrm{BP}$, before placebo a
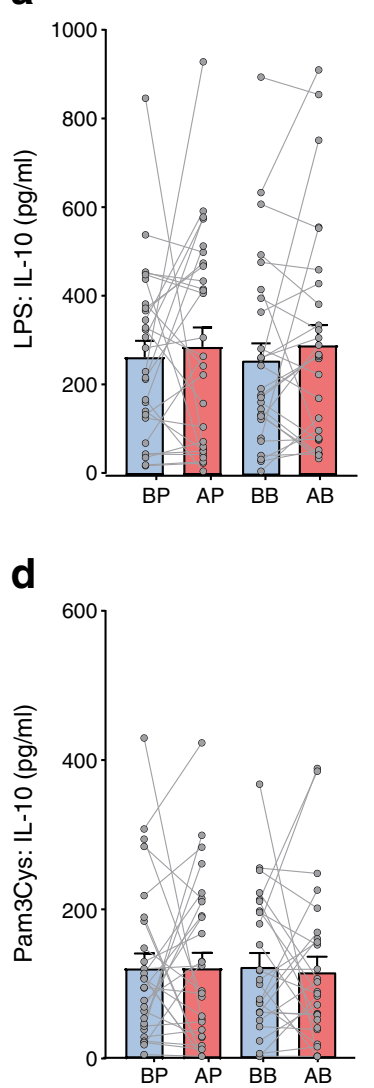

b

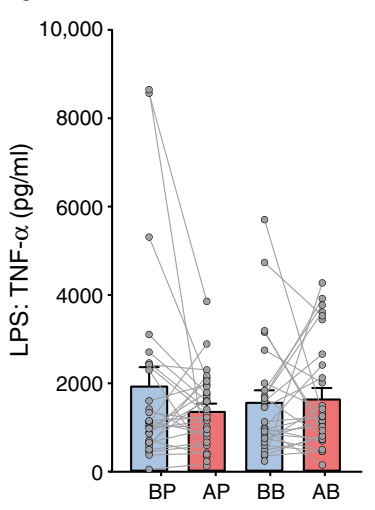

e

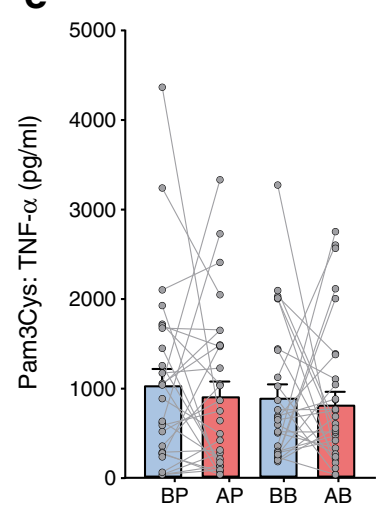

C

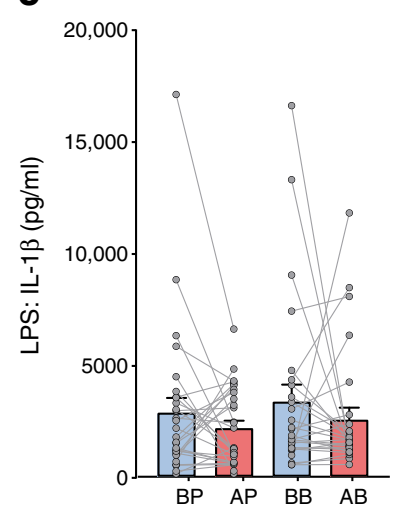

f

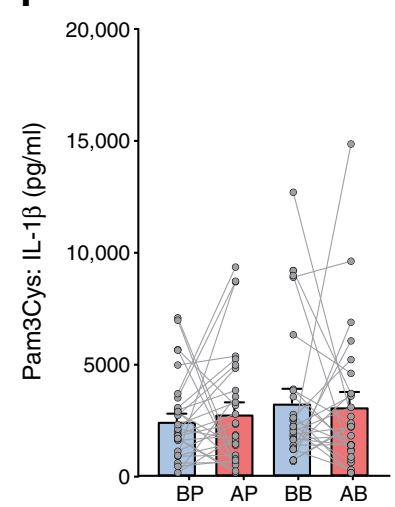

from $4.36 \pm 0.27$ to $4.33 \pm 0.34$ vs placebo, from $4.35 \pm 0.26$ to $4.38 \pm 0.25$, NS).

\section{Discussion}

Recent animal studies have suggested a causal role for altered intestinal SCFA metabolism in type 1 diabetes $[15,17,42]$. To our knowledge, we here present the first human study assessing the safety and effect of oral butyrate supplementation on immune system function and residual beta cell secretory capacity. We observed a significant decrease in faecal SCFA levels following oral butyrate treatment, with no change in innate immunity or residual beta cell function. Although we observed a change in autoreactive IA-2specific $\mathrm{CD}^{+} \mathrm{T}$ cells, this was only in a subset of $13 / 30$ participants with HLA-DR3/4 and -DQ2/8, and with large baseline variation between the groups. Thus, our data in human type 1 diabetes seem to contrast abounding literature on the beneficial effects of butyrate in mouse models of type 1 diabetes. This may be explained by differences in the mode of administration and dosing, the timing and duration of the intervention in relation to the stage of the disease process, systemic vs local effects of butyrate or differences between human and animal physiology, pathology and microbiology.
Exploring these issues may contribute to our understanding of the role of butyrate in (new-onset) human type 1 diabetes and guide future research.

Recently, butyrate and acetate supplementation were shown to protect NOD mice from developing autoimmune diabetes [15]. Diet-induced increases in butyrate affected Treg activity, while acetate decreased splenic B cells. However, type 1 diabetes in humans does not necessarily require B cells [43]. In addition, these effects were observed after a diet optimised for high colonic release of butyrate and acetate, rather than direct oral administration of SCFA, complicating direct comparison. In line with our previous study that found similar faecal bacterial strains to be altered upon oral butyrate treatment in humans [30], we observed that several species of Lachnospiraceae, a family containing many important butyrate-producing genera, were more abundant after butyrate use (L. pectinoschiza, D. formicigenerans), while Lachnospiraceae Blautia, Lachnospiraceae Marvinbryantia, Lachnospiraceae NK4A136 group and Faecalibacterium prausnitzii were less abundant. However, it should be noted that the small intestinal microbiota may be more important in type 1 diabetes pathogenesis than the faecal microbiota [44]. Thus, it is conceivable that oral butyrate supplementation downregulates the intestinal abundance of butyrate producers, or downregulates microbial gene 


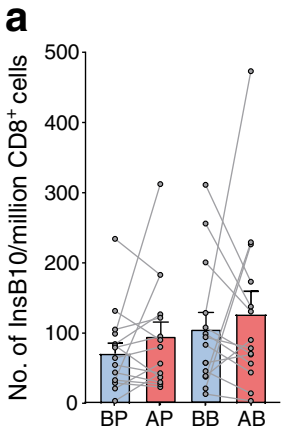

b

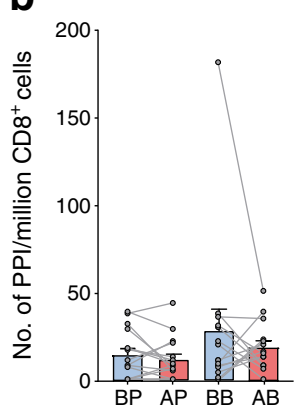

e

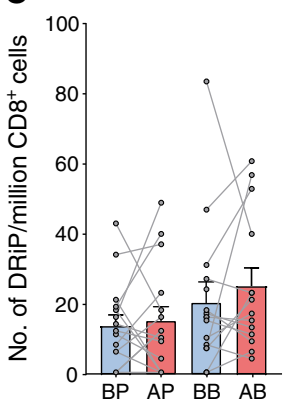

f

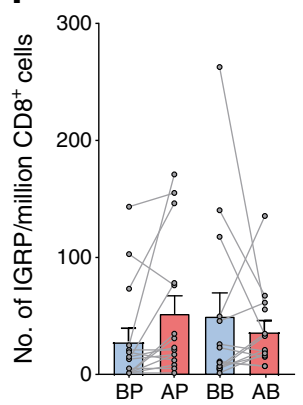

Fig. 3 Islet autoimmunity of CD8 T cells, as assessed using quantum dot multiplexed HLA-A2 tetramers loaded with a range of islet epitopes. Data are means \pm SEM, with the dots and connecting lines representing individual participants. These analyses could only be carried out on participants with HLA-DR3/4 and -DQ2/8 (13/30) and furthermore, values from one participant in the 'before placebo' group and two participants in the 'after

expression involved in the production of butyrate, as butyrate is a waste product of these microbes. This corresponds to our finding of lower faecal total SCFA and butyrate levels upon oral butyrate supplementation. It is therefore possible that oral butyrate supplementation is counterproductive, whereas supplementation of dietary fibres would increase the abundance of intestinal butyrate-producing species and associated benefits.

$\mathrm{T}$ cell autoimmunity in individuals with type 1 diabetes persists decades after diagnosis, which has been demonstrated in individuals with type 1 diabetes who received either full
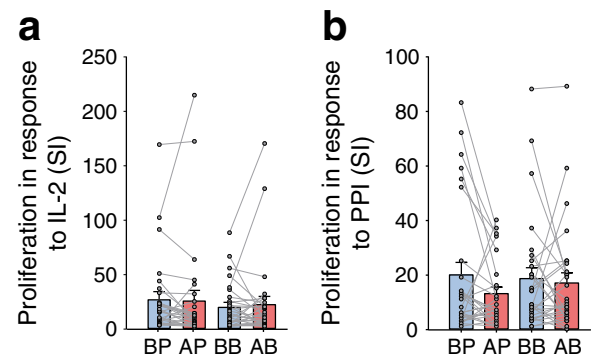

Fig. $4 \mathrm{~T}$ cell proliferation in response to islet autoantigens, expressed as lymphocyte stimulation index, which is obtained by dividing the median proliferation of three measurements of the number of stimulated cells by the median of three cell counts of unstimulated cells (medium control). Data are
C

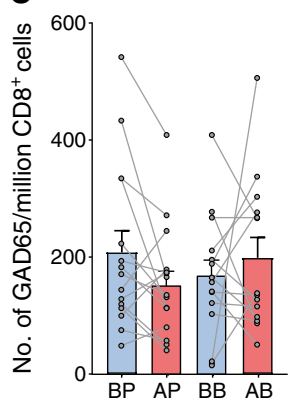

g

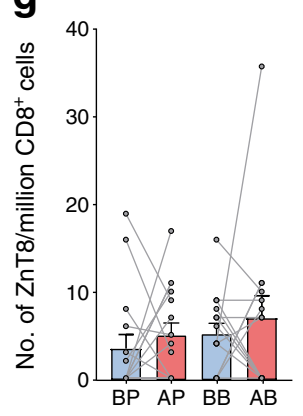

d

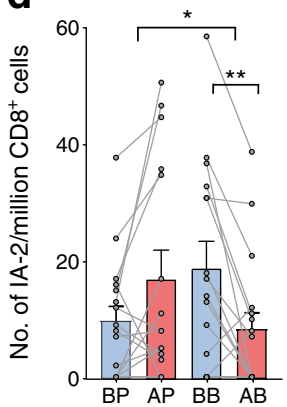

h

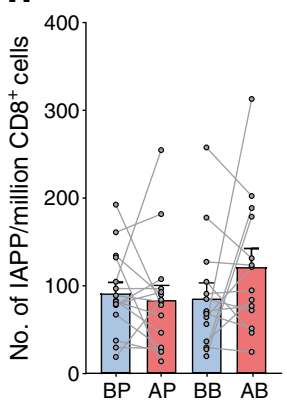

butyrate' group are missing; therefore this analysis is based on 12/30 placebo and 11/30 butyrate. Paired two-sided Wilcoxon's ranked sum tests were used to assess significance: $* p<0.05, * * p<0.01$. AB, after butyrate; $\mathrm{AP}$, after placebo; BB, before butyrate; BP, before placebo; IAPP, islet amyloid polypeptide; IGRP, islet-specific glucose-6-phosphatase catalytic subunit-related protein; InsB10, insulin B chain peptide 10; $\mathrm{ZnT} 8$, zinc transporter 8

pancreas transplants [24] or islet transplants. In these individuals, a recurrence of islet autoimmunity can be seen [45-47]. Our study showed no relevant effect of oral butyrate on T cell autoimmunity. Inherent to clinical studies, we could only investigate immune cells in peripheral blood. In mouse studies, differences in splenic B cells and Treg cells were observed that were not reflected in the pancreatic and mesenteric lymph nodes, underscoring important compartmental differences in characterising immune phenotype and function, although peripheral islet autoreactive $T$ cells have been shown to mimic islet autoimmunity in pancreatic lesions [23, 47-49]. While
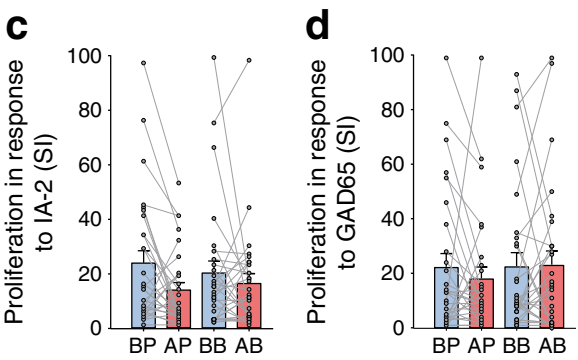

means \pm SEM, with the dots and connecting lines representing individual study participants. All participants were included in this analysis. $\mathrm{AB}$, after butyrate; AP, after placebo; BB, before butyrate; BP, before placebo; SI, stimulation index 
Fig. 5 Lymphocyte subset flow cytometry analysis. Cell numbers are expressed as total number of cells. Data are reported as the median with IQR, and whiskers show minimum and maximum values (other than outliers, shown as dots). All participants were included in this analysis. AB, after butyrate; AP, after placebo; $\mathrm{BB}$, before butyrate; $\mathrm{BP}$, before placebo; nTreg, natural Treg a

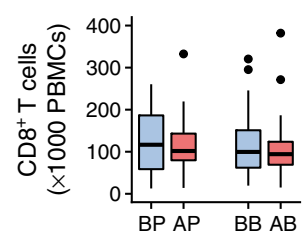

d

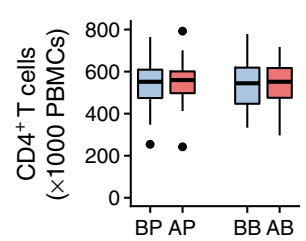

g

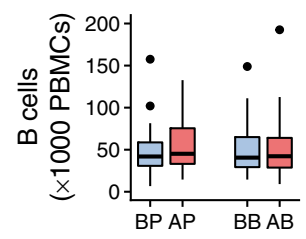

b

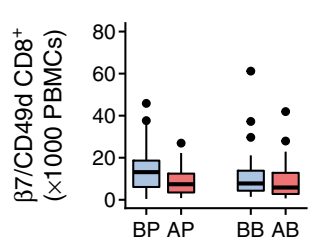

e

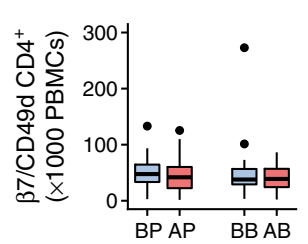

h

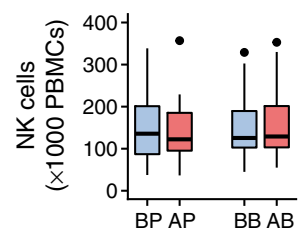

C

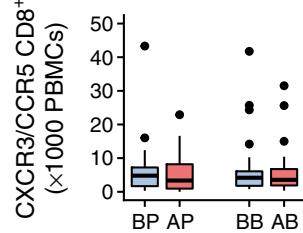

f

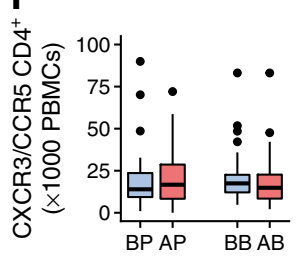

i

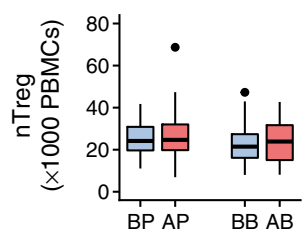

these changes may be mediated by changes in intestinal epithelial integrity, as seen in mouse models [50], we did not observe an increase in the intestinal inflammation marker faecal calprotectin.
Fig. 6 Glucose regulation, inflammation and SCFAs. (a) Stimulated C-peptide in mixedmeal test (sampling at $t=0$ and $t=90 \mathrm{~min}$ after meal challenge). (b) $\mathrm{HbA}_{1 \mathrm{c}}$. (c) Daily insulin use per $\mathrm{kg}$ of body weight. (d) CRP. (e) Faecal calprotectin. (f) Faecal levels of total SCFAs. (g) Acetate. (h) Propionate. (i) Butyrate. Data are reported as medians with IQR, and whiskers show minimum and maximum values (other than outliers, shown as dots). Paired two-sided Wilcoxon's ranked sum tests were used to assess significance: $* p<0.05$,

$* * p<0.01$. All participants were included in this analysis. $\mathrm{AB}$, after butyrate; AP, after placebo; $\mathrm{BB}$, before butyrate; $\mathrm{BP}$, before placebo
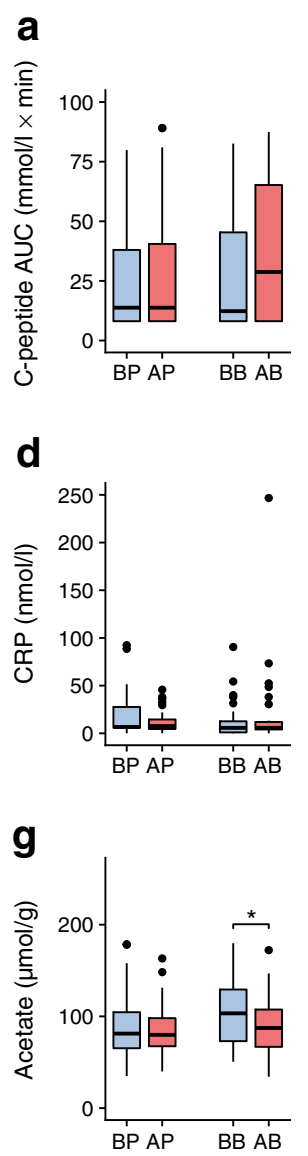

b

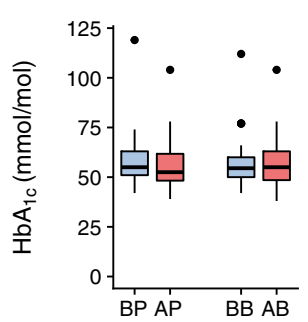

e

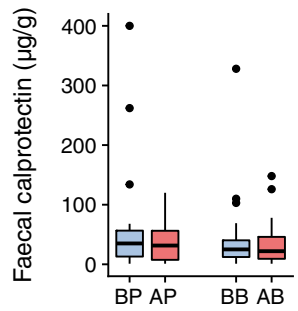

h

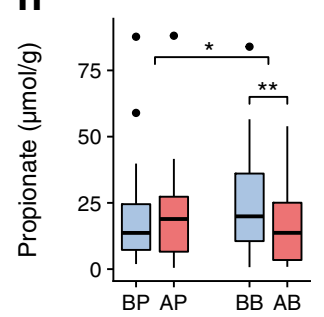

C

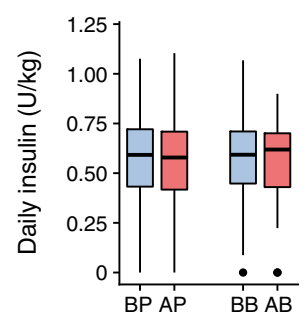

f

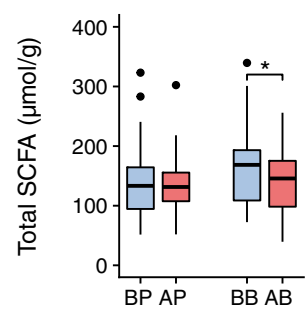

i

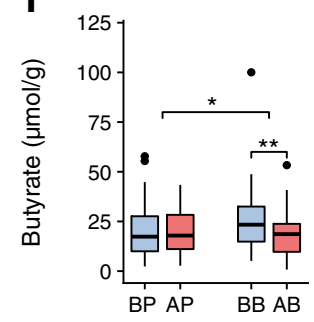


Fig. 7 Faecal microbiota composition upon butyrate supplementation. (a) Spider plot showing the relative microbial abundances of the top ten selected variables (microbial taxa) that discriminate best between butyrate and placebo use. (b) Stacked bar chart showing the relative abundance of the top ten discriminating taxa. (c) Partial least-squares discriminant analysis plot of the ten most discriminative features (microbial taxa), where coordinates on two principal components (PCs) are plotted for relative differences of microbial abundances on the butyrate and placebo groups. Incomplete separation is observed. All participants were included in this analysis. AB, after butyrate; AP, after placebo; $\mathrm{BB}$, before butyrate; $\mathrm{BP}$, before placebo

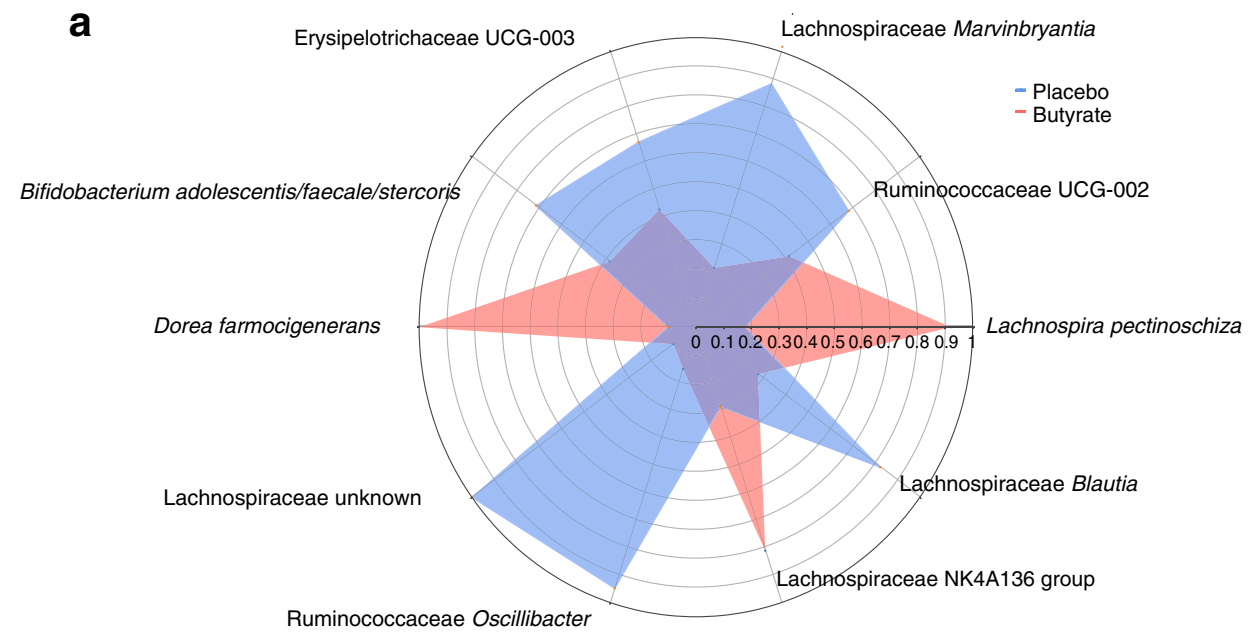

b

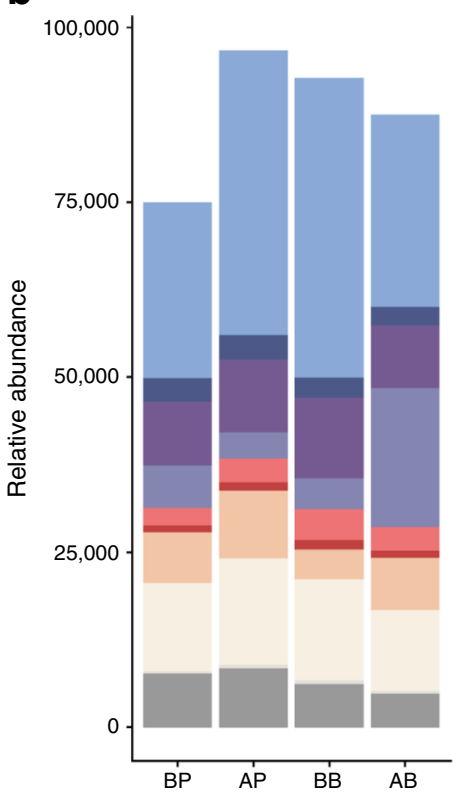

C

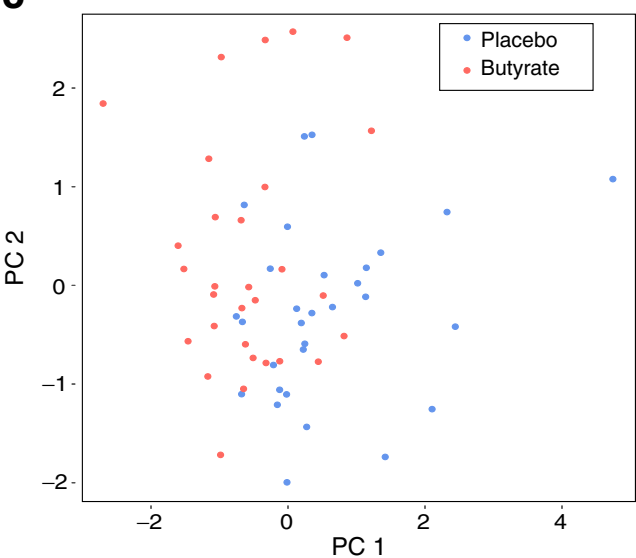

Taxon

Bifidobacterium adolescentis/faecale/stercoris

Dorea farmocigenerans

Erysipelotrichaceae UCG-003

Lachnospira pectinoschiza

Lachnospiraceae Blautia

Lachnospiraceae Marvinbryantia

Lachnospiraceae NK4A136 group

Lachnospiraceae unknown

Ruminococcaceae Oscillibacter

Ruminococcaceae UCG-002
Our study was conducted in participants with longstanding type 1 diabetes and not in those with new-onset type 1 diabetes, which would be an interesting treatment group for future clinical studies. In line with our previous human study [30] we used a standard daily dose of $4 \mathrm{~g}$ sodium butyrate given by the oral route to increase butyrate availability in the small intestine rather than in the colon, as both innate and adaptive immune cells are believed to be trained in the small intestinal mucosa before exerting their function in the peripheral tissues [51]. In addition, dysbiosis specifically in the duodenum and pancreatic ducts has been implicated in innate immune-mediated beta cell infiltration [52]. Our data suggest that treatment altered SCFA concentrations across the entire gastrointestinal tract, as faecal amounts were decreased. Thus, the current mode of delivery should be sufficient to affect training of immune cells involved in beta cell destruction and inflammation. Nevertheless, rectal SCFA administration also warrants consideration, as rectal butyrate administration at roughly $12.5 \%$ of our oral dose in humans has already been shown to achieve increased but safe butyrate levels in portal venous, hepatic venous and peripheral blood [53]. Finally, we chose a crossover design so that participants could be their own reference and to increase statistical power, as eligible individuals in the region were limited by necessarily strict inclusion criteria. Although we have addressed a potential carry-over effect in our statistical analysis, our crossover design may impair assessment of immunological memory variables such as trained immunity [54].

In conclusion, in this first-in-humans study, we report that a course of oral butyrate supplementation in individuals with 
longstanding type 1 diabetes was safe, but did not affect peripheral blood innate and adaptive immunity in any of our assays. This puts into perspective prior research on the promising role of butyrate as an immune regulator in human type 1 diabetes. Nevertheless, future studies should elucidate whether butyrate can benefit individuals with new-onset type 1 diabetes or their IA-positive siblings if a longer treatment duration and/or different modes of directly or indirectly increasing intestinal butyrate levels are used.

Acknowledgements We cordially thank C. Rustemeijer (Amstelland Hospital, Amstelveen, the Netherlands), V. Gerdes (Amsterdam UMC, Amsterdam, the Netherlands), T. Brouwer (OLVG Hospital, Amsterdam, the Netherlands), S. van Dam (OLVG Hospital, Amsterdam, the Netherlands) and J. Hensbergen (Amsterdam UMC, Amsterdam, the Netherlands) for inclusion of participants.

Data availability The datasets generated and/or analysed during the current study are available from the corresponding author on reasonable request. The full trial protocol is available from the corresponding author upon request. Raw sequencing data are available in the European Nucleotide Archive repository (https://www.ebi.ac.uk/ena/browse) under study PRJEB30292.

Funding This study was supported by Le Ducq consortium grant 17CVD01 (to $\mathrm{MNi}$ ) and CVON grant $2 \mathrm{O} 18.27$ (to $\mathrm{SB}, \mathrm{MNe}$ and $\mathrm{MNi}$ ). $\mathrm{MNi}$ is supported by a personal ZONMW-VIDI grant 2013 (016.146.327) and a Dutch Heart Foundation grant.

Duality of interest The authors declare that there is no duality of interest associated with this manuscript.

Contribution statement PFdeG, FH, JBLH, BOR and MNi designed the study. SS substantially contributed to the acquisition of data. PFdeG, BOR, MNe, ESGS, FMK, DHvR, TN, SB, GD, HH, MW, BH, JK, EMK, JHML, SI, EL, GMD-T, MD, FAvH, RB and AB contributed to the analysis and/or interpretation of data. PFdeG, BOR and MNi drafted the manuscript. All authors critically revised the manuscript. All authors gave their approval of the final (published) version of the manuscript. All authors agree to be accountable for all aspects of the work in ensuring that questions related to the accuracy or integrity of any part of the work are appropriately investigated and resolved. PFdeG is the guarantor of this work and, as such, had full access to all the data in the study and takes responsibility for the integrity of the data and the accuracy of the data analysis.

\section{References}

1. Patterson CC, Dahlquist GG, Gyürüs E, Green A, Soltész G (2009) Incidence trends for childhood type 1 diabetes in Europe during 1989-2003 and predicted new cases 2005-20: a multicentre prospective registration study. Lancet 373(9680):2027-2033. https://doi.org/10.1016/S0140-6736(09)60568-7

2. Brown CT, Davis-Richardson AG, Giongo A et al (2011) Gut microbiome metagenomics analysis suggests a functional model for the development of autoimmunity for type 1 diabetes. PLoS One 6(10):e25792. https://doi.org/10.1371/journal.pone.0025792

3. Davis-Richardson AG, Triplett EW (2015) A model for the role of gut bacteria in the development of autoimmunity for type 1 diabetes. Diabetologia 58(7):1386-1393. https://doi.org/10.1007/ s00125-015-3614-8

4. de Goffau MC, Luopajärvi K, Knip M et al (2013) Fecal microbiota composition differs between children with $\beta$-cell autoimmunity and those without. Diabetes 62(4):1238-1244. https://doi.org/10.2337/ db12-0526

5. de Goffau MC, Fuentes S, van den Bogert B et al (2014) Aberrant gut microbiota composition at the onset of type 1 diabetes in young children. Diabetologia 57(8):1569-1577. https://doi.org/10.1007/ s00125-014-3274-0

6. Samuelsson U, Ludvigsson J (2004) The concentrations of shortchain fatty acids and other microflora-associated characteristics in faeces from children with newly diagnosed type 1 diabetes and control children and their family members. Diabet Med 21(1):64 67. https://doi.org/10.1046/j.1464-5491.2003.01066.x

7. de Groot PF, Belzer C, Aydin O et al (2017) Distinct fecal and oral microbiota composition in human type 1 diabetes, an observational study. PLoS One 12(12):e0188475. https://doi.org/10.1371/journal. pone. 0188475

8. Russo I, Luciani A, De Cicco P, Troncone E, Ciacci C (2012) Butyrate attenuates lipopolysaccharide-induced inflammation in intestinal cells and Crohn's mucosa through modulation of antioxidant defense machinery. PLoS One 7(3):e32841. https://doi.org/ 10.1371/journal.pone.0032841

9. Cleophas MCP, Ratter JM, Bekkering S et al (2019) Effects of oral butyrate supplementation on inflammatory potential of circulating peripheral blood mononuclear cells in healthy and obese males. Sci Rep 9(1):775. https://doi.org/10.1038/s41598-018-37246-7

10. Arpaia N, Campbell C, Fan X et al (2013) Metabolites produced by commensal bacteria promote peripheral regulatory $\mathrm{T}$ cell generation. Nature 504(7480):451-455. https://doi.org/10.1038/ nature 12726

11. Furusawa Y, Obata Y, Fukuda S et al (2013) Commensal microbederived butyrate induces the differentiation of colonic regulatory $\mathrm{T}$ cells. Nature 504(7480):446-450. https://doi.org/10.1038/ nature 12721

12. Zimmerman MA, Singh N, Martin PM et al (2012) Butyrate suppresses colonic inflammation through HDAC1-dependent Fas upregulation and Fas-mediated apoptosis of T cells. Am J Physiol Gastrointest Liver Physiol 302(12):G1405-G1415. https://doi.org/ 10.1152/ajpgi.00543.2011

13. Jaakkola I, Jalkanen S, Hänninen A (2003) Diabetogenic T cells are primed both in pancreatic and gut-associated lymph nodes in NOD mice. Eur J Immunol 33(12):3255-3264. https://doi.org/10.1002/ eji.200324405

14. Endesfelder D, Engel M, Davis-Richardson AG et al (2016) Towards a functional hypothesis relating anti-islet cell autoimmunity to the dietary impact on microbial communities and butyrate production. Microbiome 4(1):17. https://doi.org/10.1186/s40168016-0163-4

15. Marino E, Richards JL, McLeod KH et al (2017) Gut microbial metabolites limit the frequency of autoimmune $\mathrm{T}$ cells and protect against type 1 diabetes. Nat Immunol 18(5):552-562. https://doi. org/10.1038/ni.3713

16. Markle JGM, Frank DN, Adeli K, von Bergen M, Danska JS (2014) Microbiome manipulation modifies sex-specific risk for autoimmunity. Gut Microbes 5(4):485-493. https://doi.org/10.4161/gmic. 29795

17. Desai MS, Seekatz AM, Koropatkin NM et al (2016) A dietary fiber-deprived gut microbiota degrades the colonic mucus barrier and enhances pathogen susceptibility. Cell 167(5):1339-1353.e21. https://doi.org/10.1016/j.cell.2016.10.043

18. Bosi E, Molteni L, Radaelli MG et al (2006) Increased intestinal permeability precedes clinical onset of type 1 diabetes. Diabetologia 49(12):2824-2827. https://doi.org/10.1007/s00125006-0465-3 
19. Devaraj S, Dasu MR, Park SH, Jialal I (2009) Increased levels of ligands of Toll-like receptors 2 and 4 in type 1 diabetes. Diabetologia 52(8):1665-1668. https://doi.org/10.1007/s00125009-1394-8

20. Chang PV, Hao L, Offermanns S, Medzhitov R (2014) The microbial metabolite butyrate regulates intestinal macrophage function via histone deacetylase inhibition. Proc Natl Acad Sci U S A 111(6):2247-2252. https://doi.org/10.1073/pnas.1322269111

21. Ohata A, Usami M, Miyoshi M (2005) Short-chain fatty acids alter tight junction permeability in intestinal monolayer cells via lipoxygenase activation. Nutrition 21(7):838-847. https://doi.org/ 10.1016/j.nut.2004.12.004

22. Wen L, Ley RE, Volchkov PY et al (2008) Innate immunity and intestinal microbiota in the development of type 1 diabetes. Nature 455(7216):1109-1113. https://doi.org/10.1038/nature07336

23. Coppieters KT, Dotta F, Amirian N et al (2012) Demonstration of islet-autoreactive CD8 T cells in insulitic lesions from recent onset and long-term type 1 diabetes patients. J Exp Med 209(1):51-60. https://doi.org/10.1084/jem.20111187

24. Oram RA, Jones AG, Besser REJ et al (2014) The majority of patients with long-duration type 1 diabetes are insulin microsecretors and have functioning beta cells. Diabetologia 57(1):187-191. https://doi.org/10.1007/s00125-013-3067-x

25. Williams GM, Long AE, Wilson IV et al (2016) Beta cell function and ongoing autoimmunity in long-standing, childhood onset type 1 diabetes. Diabetologia 59(12):2722-2726. https://doi.org/10. 1007/s00125-016-4087-0

26. Campbell-Thompson M, Fu A, Kaddis JS et al (2016) Insulitis and $\beta$-cell mass in the natural history of type 1 diabetes. Diabetes 65(3): 719-731. https://doi.org/10.2337/db15-0779

27. Netea MG, Joosten LAB, Latz E et al (2016) Trained immunity: a program of innate immune memory in health and disease. Science 352(6284):aaf1098. https://doi.org/10.1126/science.aaf1098

28. Kootte RS, Vrieze A, Holleman F et al (2012) The therapeutic potential of manipulating gut microbiota in obesity and type 2 diabetes mellitus. Diabetes Obes Metab 14(2):112-120. https:// doi.org/10.1111/j.1463-1326.2011.01483.x

29. Di Sabatino A, Morera R, Ciccocioppo R et al (2005) Oral butyrate for mildly to moderately active Crohn's disease. Aliment Pharmacol Ther 22(9):789-794. https://doi.org/10.1111/j.13652036.2005.02639.x

30. Bouter K, Bakker GJ, Levin E et al (2018) Differential metabolic effects of oral butyrate treatment in lean versus metabolic syndrome subjects. Clin Transl Gastroenterol 9(5):155. https://doi.org/10. 1038/s41424-018-0025-4

31. Moran A, Bundy B, Becker DJ et al (2013) Interleukin-1 antagonism in type 1 diabetes of recent onset: two multicentre, randomised, double-blind, placebo-controlled trials. Lancet 381(9881):1905-1915. https://doi.org/10.1016/S0140-6736(13) 60023-9

32. Lachin JM, McGee PL, Greenbaum CJ et al (2011) Sample size requirements for studies of treatment effects on beta-cell function in newly diagnosed type 1 diabetes. PLoS One 6(11):e26471. https:// doi.org/10.1371/journal.pone.0026471

33. van der Valk FM, Bekkering S, Kroon J et al (2016) Oxidized phospholipids on lipoprotein(a) elicit arterial wall inflammation and an inflammatory monocyte response in humans. Circulation 134(8):611-624. https://doi.org/10.1161/CIRCULATIONAHA. 116.020838

34. Velthuis JH, Unger WW, Abreu JRF et al (2010) Simultaneous detection of circulating autoreactive $\mathrm{CD} 8+\mathrm{T}$ cells specific for different islet cell-associated epitopes using combinatorial MHC multimers. Diabetes 59(7):1721-1730. https://doi.org/10.2337/ db09-1486

35. De Baere S, Eeckhaut V, Steppe M et al (2013) Development of a HPLC-UV method for the quantitative determination of four short- chain fatty acids and lactic acid produced by intestinal bacteria during in vitro fermentation. J Pharm Biomed Anal 80:107-115. https://doi.org/10.1016/j.jpba.2013.02.032

36. Salonen A, Nikkila J, Jalanka-Tuovinen J et al (2010) Comparative analysis of fecal DNA extraction methods with phylogenetic microarray: effective recovery of bacterial and archaeal DNA using mechanical cell lysis. J Microbiol Methods 81(2):127-134. https://doi.org/10.1016/j.mimet.2010.02.007

37. Ramiro-Garcia J, Hermes GDA, Giatsis C et al (2016) NG-tax, a highly accurate and validated pipeline for analysis of $16 \mathrm{~S}$ rRNA amplicons from complex biomes [version 1; referees: 2 approved with reservations, 1 not approved]. F1000Res 5:1791. https://doi. org/10.12688/f1000research.9227.1

38. Kozich JJ, Westcott SL, Baxter NT, Highlander SK, Schloss PD (2013) Development of a dual-index sequencing strategy and curation pipeline for analyzing amplicon sequence data on the MiSeq Illumina sequencing platform. Appl Environ Microbiol 79(17):5112-5120. https://doi.org/10.1128/AEM.01043-13

39. Kootte RS, Levin E, Salojarvi J et al (2017) Improvement of insulin sensitivity after lean donor feces in metabolic syndrome is driven by baseline intestinal microbiota composition. Cell Metab 26(4):611619.e6. https://doi.org/10.1016/j.cmet.2017.09.008

40. Chen T, Guestrin C (2016) XGBoost: a scalable tree boosting system. In: Proceedings of the 22nd ACM SIGKDD International Conference on Knowledge Discovery and Data Mining. ACM, New York, NY, USA, pp 785-794

41. Meinshausen N, Bühlmann P (2010) Stability selection. J R Stat Soc Series B Stat Methodol 72(4):417-473. https://doi.org/10.1111/ j.1467-9868.2010.00740.x

42. Endesfelder D, Zu Castell W, Ardissone A et al (2014) Compromised gut microbiota networks in children with anti-islet cell autoimmunity. Diabetes 63(6):2006-2014. https://doi.org/10. 2337/db13-1676

43. Martin S, Wolf-Eichbaum D, Duinkerken G et al (2001) Development of type 1 diabetes despite severe hereditary B cell deficiency. N Engl J Med 345(14):1036-1040. https://doi.org/10. 1056/NEJMoa010465

44. Badami E, Sorini C, Coccia M et al (2011) Defective differentiation of regulatory FoxP3+ T cells by small-intestinal dendritic cells in patients with type 1 diabetes. Diabetes 60(8):2120-2124. https:// doi.org/10.2337/db10-1201

45. Roep BO, Stobbe I, Duinkerken G et al (1999) Auto- and alloimmune reactivity to human islet allografts transplanted into type 1 diabetic patients. Diabetes 48(3):484-490. https://doi.org/ 10.2337/diabetes.48.3.484

46. Huurman VAL, Hilbrands R, Pinkse GGM et al (2008) Cellular islet autoimmunity associates with clinical outcome of islet cell transplantation. PLoS One 3(6):e2435. https://doi.org/10.1371/journal. pone. 0002435

47. Pinkse GGM, Tysma OHM, Bergen CAM et al (2005) Autoreactive CD8 T cells associated with beta cell destruction in type 1 diabetes. Proc Natl Acad Sci U S A 102(51):18425-18430. https://doi.org/ 10.1073/pnas.0508621102

48. Tan S, Li Y, Xia J et al (2017) Type 1 diabetes induction in humanized mice. Proc Natl Acad Sci U S A 114(41):10954-10959. https:// doi.org/10.1073/pnas.1710415114

49. Babon JAB, DeNicola ME, Blodgett DM et al (2016) Analysis of self-antigen specificity of islet-infiltrating $\mathrm{T}$ cells from human donors with type 1 diabetes. Nat Med 22(12):1482-1487. https:// doi.org/10.1038/nm.4203

50. Rouxel O, Da Silva J, Beaudoin L et al (2017) Cytotoxic and regulatory roles of mucosal-associated invariant $\mathrm{T}$ cells in type 1 diabetes. Nat Immunol 18(12):1321-1331. https://doi.org/10.1038/ni. 3854

51. Lynch L, O’Donoghue D, Dean J, O'Sullivan J, O'Farrelly C, Golden-Mason L (2006) Detection and characterization of 
hemopoietic stem cells in the adult human small intestine. $\mathrm{J}$ Immunol 176(9):5199-5204

52. Korsgren S, Molin Y, Salmela K, Lundgren T, Melhus Å, Korsgren O (2012) On the etiology of type 1 diabetes: a new animal model signifying a decisive role for bacteria eliciting an adverse innate immunity response. Am J Pathol 181(5):1735-1748. https://doi. org/10.1016/j.ajpath.2012.07.022

53. van der Beek CM, Bloemen JG, van den Broek MA et al (2015) Hepatic uptake of rectally administered butyrate prevents an increase in systemic butyrate concentrations in humans. J Nutr 145(9):2019-2024. https://doi.org/10.3945/jn.115.211193

54. David LA, Maurice CF, Carmody RN et al (2013) Diet rapidly and reproducibly alters the human gut microbiome. Nature 505(7484): 559-563. https://doi.org/10.1038/nature12820

Publisher's note Springer Nature remains neutral with regard to jurisdictional claims in published maps and institutional affiliations. 